\title{
On the Asymptotic Behaviour of First Passage Times for Discussions
}

\author{
G. Keller ${ }^{1}$, G. Kersting ${ }^{2}$, and U. Rösler ${ }^{3}$ \\ ${ }^{1}$ Institut für Angewandte Mathematik, Universität Heidelberg, Im Neuenheimer Feld 294, D-6900 \\ Heidelberg, Federal Republic of Germany \\ ${ }^{2}$ Fachbereich Mathematik, Universität Frankfurt, Robert Mayer-Straße 10, D-6000 Frankfurt, \\ Federal Republic of Germany \\ ${ }^{3}$ Institut für Mathematische Stochastik, Universität Göttingen, Lotzestraße 13, D-3400 Göttingen, \\ Federal Republic of Germany
}

Summary. We investigate the asymptotic behaviour of first passage times of diffusions or birth-death processes. Necessary and sufficient conditions in terms of moments and the speed and scale functions are given for convergence of the first passage times to a normal or an exponential distribution.

\section{Introduction}

Let $X_{t}, t \geqq 0$, be a diffusion on $\mathbb{R}^{+}$. Let $\tau_{y}$ be the passage time through some state $y>0$. We investigate the asymptotic behaviour of $\tau_{y}$, properly normalized, for $y \rightarrow \infty$. Our results extend earlier ones of Mandl (see his book (1968) for an account) and we use different methods. The distribution of $\tau_{y}$ is an object, which in general is difficult to handle. However, there is an important case, in which one has additional information: If the left boundary 0 is reflecting, and if we start in 0 , then the distribution of $\tau_{y}$ is the convolution of exponential distributions (Kac and Krein 1968). (We give a proof below.) This is the main basis of this paper and enables us to give necessary and sufficient conditions for $\tau_{y}$ to be asymptotically normal or exponential.

The correct normalization of $\tau_{y}$ is by expectation and variance:

$$
\tau_{y}^{*}:=\frac{t_{y}-E_{0} \tau_{y}}{\sqrt{\operatorname{Var}_{0} \tau_{y}}} .
$$

$\star$ Work partially supported by Deutsche Forschungsgemeinschaft, Sonderforschungsbereich 123 (Pro-
ject B2) 
The possible limits of $\tau_{y}^{*}, y \rightarrow \infty$, in distribution are convolutions of normal and exponential distributions. The important observation is that the exponential and the normal distribution are extremal elements of this class of distributions: We always have $0 \leqq E_{0}\left(\tau_{y}^{*}\right)^{3} \leqq 2$. The normal distribution is the only one with vanishing third moment, and for the exponential distribution with variance 1 the third moment is 2. It turns out: Necessary and sufficient for convergence of $\tau_{y}^{*}$ to a normal law is that the third moment of $\tau_{y}^{*}$ converges to zero as $y \rightarrow \infty$. Necessary and sufficient for convergence of $\tau_{y}^{*}$ to an exponential distribution is that the third moment of $\tau_{y}^{*}$ converges to 2 . We like to point out that from a technical point it is essential that the diffusion starts in a reflecting boundary point, but the results on the asymptotic distribution of $\tau_{y}^{*}$ also hold under different assumptions. At the beginning of Sect. 4 we explain this in the transient case, where $X_{t} \rightarrow \infty$ with probability 1 , as $t \rightarrow \infty$.

These results are stated in Sect. 3. They are consequences of a more general theorem, given in Sect. 2. There we consider an array of independent exponential distributed random variables and investigate the distribution of the sums. They are again correctly normalized by expectation and variance. Theorem 2.5 gives a series of sufficient and necessary conditions for convergence to a normal law. These include simple moment conditions and also that of asymptotical neglectibility, which is already sufficient (and of course necessary).

Coming back to diffusions, we only have to consider third moments. These are determined by the speed and scale function, which are in turn determined by the first and second moment of $\tau_{y}, y \in \mathbb{R}$. Basically (see Sect. 4 for more details) $\tau_{y}^{*}$ converges to a normal, if $\operatorname{Var}_{0} \tau_{y} \rightarrow \infty$ and $\left(\operatorname{Var}_{0} \tau_{y}\right)^{1 / 2}\left(E_{0} \tau_{y}\right)^{-1}$ converges to zero and $\tau_{y}^{*}$ converges to an exponential, if $\left(\operatorname{Var}_{0} \tau_{y}\right)^{1 / 2}\left(E_{0} \tau_{y}\right)^{-1}$ converges to 1 . Two classes of examples conclude the last section.

We did not try to extend our results to the most general processes, i.e. processes on $\mathbb{R}$ with the nearest neighbour property. One main tool is the approximation of $\left(X_{t}\right)$ by a sequence of birth and death processes, which is due to Stone (1963) for diffusions. To our knowledge the possible limits of birth and death processes are not known. For this reason we prefer to stay with (quasi) diffusions having right continuous speed function and continuous scale function.

\section{Arrays of Exponential Distributions}

A random variable $X$ has an exponential distribution with parameter $\lambda>0$, if $\operatorname{Pr}(X \geqq t)=e^{-t / \lambda}$ for $t>0$. We will also allow $\lambda \leqq 0$. The case $\lambda=0$ corresponds to $X \equiv 0$ and $\lambda<0$ to $-X$ has an exponential law with parameter $-\lambda$.

Throughout this paper $X_{k}^{n}, n \in \mathbb{N}, k \in \mathbb{Z}$ denote random variables possessing exponential distributions with parameter $\lambda_{k}^{n} \in \mathbb{R}$. For fixed $n \in \mathbb{N}$ the random variables $X_{k}^{n}, k \in \mathbb{Z}$, are independent. The first task is to find the correct normalization of $S_{n}=\sum_{k \in \mathbb{Z}} X_{k}^{n}$, i.e. suitable $a_{n}, b_{n} \in \mathbb{R}, a_{n}>0$, such that

$$
S_{n}^{*}=\frac{S_{n}-b_{n}}{a_{n}}
$$


converges vaguely. The proper normalization (neglecting trivial modifications of $a_{n}$ and $b_{n}$ ) results when $a_{n}^{2}=\operatorname{Var} S_{n}$ and $b_{n}=E S_{n}$. This is a consequence of the following proposition.

Denote by $\mathbf{L}(X)$ the distribution of a random variable $X$.

Proposition 2.1. Let $\mathbf{L}\left(S_{n}^{*}\right)$ converge vaguely as $n \rightarrow \infty$. Then either $\mathbf{L}\left(S_{n}^{*}\right)$ converges to the zero measure or $S_{n}^{*}$ converges in distribution. In the second case all moments of $S_{n}^{*}$ have a finite limit, namely the corresponding moment of the limit law.

Proof. By choosing a subsequence, we may assume that both

$$
\begin{aligned}
E S_{n}^{*} & =\sum_{k} \lambda_{k}^{n} / a_{n}-b_{n} / a_{n}, \\
\operatorname{Var} S_{n}^{*} & =\sum_{k}\left(\lambda_{k}^{n} / a_{n}\right)^{2}
\end{aligned}
$$

converge, possibly to $\pm \infty$. If $E S_{n}^{*} \rightarrow \pm \infty$ and $\operatorname{Var} S_{n}^{*}$ approaches a finite limit, an easy application of Tschebyscheff's inequality shows that $\mathbf{L}\left(S_{n}^{*}\right)$ converges to the zero measure. For the case $\operatorname{Var} S_{n}^{*} \rightarrow \infty$ check the formula

$$
\left|\varphi_{n}(t)\right|^{2}=\prod_{k}\left(1+t^{2}\left(\lambda_{k}^{n} / a_{n}\right)^{2}\right)^{-1},
$$

where $\varphi_{n}(t)=E\left(\exp\right.$ it $\left.S_{n}^{*}\right)$ is the characteristic function of $S_{n}^{*}$. $\operatorname{Var} S_{n}^{*} \rightarrow \infty$ implies $\left|\varphi_{n}(t)\right|^{2} \rightarrow 0$ for every $t \neq 0$, and by a theorem of Chung (1968), p. 152, $\mathbf{L}\left(S_{n}^{*}\right)$ again converges to the zero measure.

Next suppose that $E S_{n}^{*}$ and $\operatorname{Var} S_{n}^{*}$ converge to a finite limit. Without loss $E S_{n}^{*}=0, \operatorname{Var} S_{n}^{*}=1$ for all $n \geqq 1$. In this case $\mathbf{L}\left(S_{n}^{*}\right)$ forms a tight sequence of probability measures and we have convergence in distribution. Since we are only dealing with exponential distributions, for suitable $C_{k}, D_{k}>0, k \geqq 1$,

$$
\begin{aligned}
E\left(S_{n}^{*}\right)^{2 k} & \leqq C_{k} a_{n}^{-2 k} \sum_{k} E\left(X_{k}^{n}-E X_{k}^{n}\right)^{2 k} \\
& \leqq D_{k} \sum_{k}\left(\lambda_{k}^{n} / a_{n}\right)^{2 k} \leqq D_{k} \sum_{k}\left(\lambda_{k}^{n} / a_{n}\right)^{2}=D_{k}
\end{aligned}
$$

It is a familiar fact that this uniform bound implies the convergence of all moments to those of the limit law.

From now we shall normalize $S_{n}$ with $b_{n}=E S_{n}, a_{n}^{2}=\operatorname{Var} S_{n} \neq 0$, thus

$$
S_{n}^{*}=\frac{S_{n}-E S_{n}}{\sqrt{\operatorname{Var} S_{n}}}
$$

Next we determine the class of non-degenerate limits of $S_{n}^{*}$. It contains all exponentials and, in view of the central limit theorem, the normal. These two types comprise basically all extreme cases. All other possible limits are convolutions of these. 
Let $E$ be the set of characteristic functions

$$
\psi(t)=\psi\left(t ; \gamma,\left(\mu_{k}\right)\right)=e^{-\gamma / 2 t^{2}} \prod_{k \in \mathbb{Z}} e^{-i t \mu_{k}}\left(1-i t \mu_{k}\right)^{-1},
$$

with $\gamma \geqq 0, \mu_{k} \in \mathbb{R}, \gamma+\sum_{k} \mu_{k}^{2}=1$. By relabeling we may assume

$$
\mu_{0} \geqq \mu_{1} \geqq \ldots \geqq 0, \quad \mu_{-1} \leqq \mu_{-2} \leqq \ldots \leqq 0 .
$$

Let $E^{+}$consist of all $\psi \in E$ with the additional restriction $\mu_{k} \geqq 0, k \in \mathbb{Z} . E$ is the set of characteristic functions of the so-called Polya-frequency densities (cf. Karlin 1968, Chap. 7).

Theorem 2.2. Let $\psi_{n}(t)=\psi\left(t ; \gamma_{n},\left(\mu_{k}^{n}\right)\right)$ belong to $E\left(E^{+}\right)$. Necessary and sufficient for the pointwise convergence of $\psi_{n}(t)$ to some characteristic function $\psi(t)$ is the convergence $\mu_{k}^{n} \longrightarrow{ }_{n}$ for all $k \in \mathbb{Z}$ and suitable $\mu_{k} \in \mathbb{R}$. In this case

with

$$
\begin{gathered}
\psi(t)=\psi\left(t ; \gamma,\left(\mu_{k}\right)\right) \\
\gamma=1-\sum_{k}\left(\mu_{k}\right)^{2} .
\end{gathered}
$$

Note. In general $\gamma_{n} \nrightarrow \gamma$.

Proof. First assume $\mu_{k}^{n} \rightarrow \mu_{k}$ for all $k \in \mathbb{Z}$. Without loss assume that $\gamma_{n}$ converges to some number $\rho \geqq 0$. Let $N=N_{n}$ be a sequence of natural numbers, going to infinity

$$
\begin{aligned}
\psi_{n}(t) & =\left[e^{-\gamma_{n} t^{2} / 2} \prod_{k=-N}^{N} e^{-i t \mu_{k}^{n}}\left(1-i t \mu_{k}^{n}\right)^{-1}\right] \times\left[\prod_{|k|>N} e^{-i t \mu_{k}^{n}}\left(1-i t \mu_{k}^{n}\right)^{-1}\right] \\
& =\psi_{n 1}(t) \psi_{n 2}(t) \quad \text { (say). }
\end{aligned}
$$

If $N_{n} \rightarrow \infty$ slowly enough

$$
\psi_{n 1}(t) \underset{n}{\longrightarrow} e^{-\rho t^{2} / 2} \prod_{k=-\infty}^{\infty} e^{-i t \mu_{k}}\left(1-i t \mu_{k}\right)^{-1} .
$$

Further, using $\ln (1+x)=x-x^{2} / 2+R(x)$ with $|R(x)| \leqq|x|^{3}(1-|x|)^{-1}$ for $|x|<1$,

$$
\begin{aligned}
\ln \psi_{n 2}(t) & =\sum_{|k|>N}\left(-i t \mu_{k}^{n}-\ln \left(1-t i \mu_{k}^{n}\right)\right) \\
& =-t^{2} / 2 \sum_{|k|>N}\left(\mu_{k}^{n}\right)^{2}-\sum_{|k|>N} R\left(-i t \mu_{k}^{n}\right) .
\end{aligned}
$$

Now, if $N \rightarrow \infty$ slowly enough,

$$
\sum_{|k|>N}\left(\mu_{k}^{n}\right)^{2}=1-\gamma_{n}-\sum_{|k|>N}\left(\mu_{k}^{n}\right)^{2} \underset{n}{\longrightarrow} 1-\rho-\sum_{k=-\infty}^{\infty}\left(\mu_{k}\right)^{2},
$$


and

$$
\left|\sum_{|k|>N} R\left(i t \mu_{k}^{n}\right)\right| \leqq \sum_{k}\left(\mu_{k}^{n}\right)^{2} \sup _{|k|>N} \frac{\left|\mu_{k}^{n}\right|}{1-\left|\mu_{k}^{n}\right|} \leqq \frac{\mu_{N}^{n}}{1-\mu_{N}^{n}}+\frac{-\mu_{-N}^{n}}{1+\mu_{N}^{n}} \underset{n}{\longrightarrow} 0 .
$$

Thus

$$
\psi_{n 2}(t) \underset{n}{\longrightarrow} \exp \left(-t^{2} / 2\left(1-\rho-\sum_{k}\left(\mu_{k}\right)^{2}\right)\right)=\exp \left(-t^{2} / 2(\gamma-\rho)\right),
$$

and one direction of the statement follows. For the other one note that $\psi\left(t ; \gamma, \mu_{k}\right)$, $t \in \mathbb{R}$, uniquely determines $\mu_{k}$ and $\gamma:-i / \mu_{k}$ are exactly the poles of its complex continuation, counted with their multiplicities. Thus, if $\mu_{k}^{n}$ does not converge for some $k \in \mathbb{Z}$, by a diagonalization argument we can find two pointwise convergent subsequences of $\psi_{n}(t)$ with different limits.

As a consequence of the theorem we note the well-known fact that $E$ and $E^{+}$are closed under convergence in distribution (cf. Karlin 1968, p. 340). In probabilistic language:

Corollary 2.3. Let the distribution of $S_{n}^{*}$ converge to some probability law $F$. Then $F$ is the convolution of a normal and exponential distributions. If $S_{n} \geqq 0$, then $F$ is the convolution of a normal and exponential distributions with non-negative parameters.

To apply Theorem 2.2 to $S_{n}^{*}$, it is in general necessary to know the $\lambda_{k}^{n}$. However, in the most important situations it is sufficient to know the asymptotic behaviour of the third and fourth moment of $S_{n}^{*}$.

Proposition 2.4. It holds

$$
\begin{aligned}
& E S_{n}^{*}=\sum_{k} \frac{\lambda_{k}^{n}}{a_{n}}-\frac{b_{n}}{a_{n}}=0, \\
& \operatorname{Var} S_{n}^{*}=\sum_{k}\left(\lambda_{k}^{n} / a_{n}\right)^{2}=1, \\
& E\left(S_{n}^{*}-E S_{n}^{*}\right)^{3}=2 \sum_{k}\left(\lambda_{k}^{n} / a_{n}\right)^{3}, \\
& E\left(S_{n}^{*}-E S_{n}^{*}\right)^{4}=3+6 \sum_{k}\left(\lambda_{k}^{n} / a_{n}\right)^{4} .
\end{aligned}
$$

Proof. Simple computation.

Now we shall give necessary and sufficient conditions on $\lambda_{k}^{n}$ for the convergence to an $\mathbf{N}(0,1)$-distribution. Comparing this with general results, we know that

$$
Y_{k}^{n}=\frac{X_{k}^{n}-E X_{k}^{n}}{\sqrt{\operatorname{Var} S_{n}}}
$$

must be asymptotically neglectible, i.e.

$$
\sup _{k} \operatorname{Pr}\left(\left|Y_{k}^{n}\right| \geqq \varepsilon\right) \underset{n}{\longrightarrow} 0
$$

for all $\varepsilon>0$. In fact this condition is also necessary. 
Theorem 2.5. The following is equivalent:

(i) $\mathbf{L}\left(S_{n}^{*}\right) \longrightarrow \mathbf{N}(0,1)$

(ii) $\sup _{k}\left|\lambda_{k}^{n} / a_{n}\right| \underset{n}{\longrightarrow} 0$,

(iii) $\sum_{k}\left|\lambda_{k}^{n} / a_{n}\right|^{r} \underset{n}{\longrightarrow} 0$ for some $r>2$,

(iv) $E\left(S_{n}^{*}\right)^{4} \longrightarrow 3$

(v) $\sup _{k} \operatorname{Pr}\left(\left|Y_{k}^{n}\right|>\varepsilon\right) \rightarrow 0$ for all $\varepsilon>0$,

(vi) $\sum_{k} \operatorname{Pr}\left(\left|Y_{k}^{n}\right|>\varepsilon\right) \rightarrow 0$ for all $\varepsilon>0$,

(vii) $\operatorname{Pr}\left(\sup _{k}\left|Y_{k}^{n}\right|>\varepsilon\right) \rightarrow 0$ for all $\varepsilon>0$.

Proof. (i) $\Leftrightarrow$ (ii) From Theorem 2.2 with $\mu_{k}^{n}=\lambda_{k}^{n} / a_{n}$ after a suitable rearrangement of the $X_{k}^{n}$.

(ii) $\begin{aligned} \Leftrightarrow \text { (iii) } \sup _{k}\left|\lambda_{k}^{n} / a_{n}\right|^{r} \leqq \sum_{k}\left|\lambda_{k}^{n} / a_{n}\right|^{r} & \leqq \sup _{k}\left|\lambda_{k}^{n} / a_{n}\right|^{r-2} \sum_{k}\left(\lambda_{k}^{n} / a_{n}\right)^{2} \\ & =\sup _{k}\left|\lambda_{k}^{n} / a_{n}\right|^{r-2} .\end{aligned}$

(iii) $\Leftrightarrow$ (iv) Use Proposition 2.4.

(ii) $\Leftrightarrow$ (v) $\Leftrightarrow$ (vi) $\Leftrightarrow$ (vii) Simple computation, since $X_{k}^{n}$ is exponential.

Corollary 2.6. If $S_{n} \geqq 0$, then $\mathbf{L}\left(S_{n}^{*}\right) \underset{n}{\longrightarrow} \mathbf{N}(0,1)$, iff the third moment of $S_{n}^{*}$ converges to zero.

Proof. Here $\lambda_{k}^{n} \geqq 0$ for all $k, n$. Use Proposition 2.4 and Theorem 2.5 iv).

Corollary 2.7. If $S_{n} \geqq 0$, then $S_{n}^{*}$ is asymptotically normal, if it converges in law to a symmetric distribution.

Corollary 2.8. If $S_{n} \geqq 0$, then $\mathbf{L}\left(S_{n}^{*}\right) \underset{n}{\longrightarrow} \mathbf{N}(0,1)$ entails $\sqrt{\operatorname{Var} S_{n}}=o\left(E S_{n}\right)$ as $n \rightarrow \infty$.

Proof. Without loss $a_{n}=1$. Then from Theorem 2.5

$$
\sqrt{\operatorname{Var} S_{n}}=1=\sum_{k}\left(\lambda_{k}^{n}\right)^{2} \leqq \sup _{k}\left|\lambda_{k}^{n}\right| \sum_{k} \lambda_{k}^{n}=o\left(E S_{n}\right)
$$

Next we consider the case of an exponential limit.

Theorem 2.9. The following is equivalent:

(i) $\mathbf{L}\left(S_{n}^{*}\right)$ converges to an exponential limit (centered at expectation),

(ii) $\sup _{k}\left|\lambda_{k}^{n} / a_{n}\right| \underset{n}{\longrightarrow} 1$,

(iii) $\sum_{k}\left|\lambda_{k}^{n} / a_{n}\right|^{r} \underset{n}{\longrightarrow} 1$ for some $r>2$,

(iv) $E\left(S_{n}^{*}\right)^{4} \underset{n}{\longrightarrow} 9$. 
Proof. (i) $\Leftrightarrow$ (ii) Apply Theorem 2.2, where again we may assume $\mu_{k}^{n}=\lambda_{k}^{n} / a_{n}$. Note $\sup _{k}\left|\lambda_{k}^{n} / a_{n}\right|=\max \left\{\mu_{0}^{n},-\mu_{-1}^{n}\right\}$. We have an exponential limit, iff $\mu_{0}^{n} \rightarrow 1$ or $\mu_{-1}^{n} \rightarrow$ -1 .

(ii) $\Leftrightarrow$ (iii), (iii) $\Leftrightarrow$ (iv) as above.

Corollary 2.10. If $S_{n} \geqq 0$, then $\mathbf{L}\left(S_{n}^{*}\right)$ is asymptotically exponential, iff $E\left(S_{n}^{*}\right)^{3} \underset{n}{\longrightarrow}$.

Corollary 2.11. If $S_{n} \geqq 0$ and $\sqrt{\operatorname{Var} S_{n}} / E S_{n} \longrightarrow$, then $\mathbf{L}\left(S_{n}^{*}\right)$ converges to an exponential law.

Proof. Without loss $a_{n}=1$. If along a subsequence $\sup _{k}\left|\lambda_{k}^{n}\right| \leqq c<1$,

$$
\sqrt{\operatorname{Var} S_{n}}=1=\sum_{k}\left(\lambda_{k}^{n}\right)^{2} \leqq c \sum_{k} \lambda_{k}^{n}=c E S_{n}
$$

which contradicts the assumption.

\section{Diffusions}

Let $X_{t}, t \geqq 0$, be a strong Markov process with state space $S \subset \mathbb{R}$. Such a process is called regular, if for all starting points $x \in \operatorname{int}(S)$ and all $y \in S P_{x}\left(\tau_{y}<\infty\right)>0$. $\tau_{y}$ is the first hitting time of $y$, i.e.

$$
\tau_{y}=\inf \left\{t: X_{t}=y\right\} .
$$

A diffusion process is a regular strong Markov process with continuous paths and state space $\mathbb{R}$. To such a diffusion corresponds a semigroup $T_{t}: C_{0} \rightarrow C_{0}$, $t \geqq 0$, by

$$
\begin{gathered}
T_{t} f(x)=E_{x} f\left(X_{t}\right), \\
C_{0}:=\left\{f: \mathbb{R} \rightarrow \mathbb{R} \text { continuous, } \lim _{|x| \rightarrow \infty} f(x)=0\right\} .
\end{gathered}
$$

The infinitesimal operator $L$, defined by

$$
L f(x)=\lim _{t \rightarrow 0} \frac{T_{t} f(x)-f(x)}{t},
$$

whenever the right side makes sense, is of the form of a second-order differential operator of Feller type $D_{m} D_{p}^{+}$, although the domain of definition might be different. Here $m, p: \rightarrow \mathbb{R}$ are strictly monoton increasing functions, $m$ right continuous, $p$ continuous. The operators $D_{m}, D_{p}^{+}$are defined by

$$
D_{m} f(x)=\lim _{y \rightarrow x} \frac{f(y)-f(x)}{m(y)-m(x)}, \quad D_{p}^{+} f(x)=\lim _{y \downarrow x} \frac{f(y)-f(x)}{p(y)-p(x)}
$$


on $C_{0}$, if the right side gives sense. (See Feller (1959) or Mandl (1968) for a detailed version.) Further the boundaries $\pm \infty$ are assumed to be natural, i.e.

$$
\int_{0}^{\infty} \int_{0}^{y} d m(z) d p(y)=\int_{-\infty}^{0} \int_{y}^{0} d m(z) d p(y)=\infty .
$$

By this condition the process $X_{t}$ has infinite lifetime and never reaches the boundary. $m$ is called the speed function, $p$ the scale function. On the other hand every $m$ and $p$ as above, including the boundary conditions, provide by

$$
T_{t}=\exp t D_{m} D_{p}^{+}, \quad t \geqq 0
$$

a strongly continuous semigroup and hence a diffusion.

In the sequel we shall consider diffusions on $\mathbb{R}^{+}$, having a reflecting boundary zero. A formal way to introduce them avoiding any boundary terminology is the following. Let $X_{t}$ be a diffusion on $\mathbb{R}$ with speed function $m$, scale function $p$. Assume $p(x)=-p(-x), m(x)=-m(-x)$ for every continuity point $x \in \mathbb{R}$ and additionally $0=\lim _{x \rightarrow 0} m(x)=m(0)$. Then $\left|X_{t}\right|$ is a diffusion on $\mathbb{R}^{+}$with a reflecting boundary 0 and speed, scale measure $m, p$ restricted to $R^{+}$.

A birth and death process (BAD) is a strong Markov process with a discrete (countable) state space $S \subset \mathbb{R}$, for which only jumps to the next neighbours are allowed, i.e. $S$ is ordered $\ldots x_{-1}<x_{0}<x_{1}<\ldots$ then (starting in $x_{n}$ )

$\operatorname{Pr}_{x_{n}}\left(\left\{\forall t X_{t}>x_{n+1} \exists 0<s<t X_{s}=x_{n+1}\right\} \cap\left\{\forall t X_{t}<x_{n-1} \exists 0<s<t X_{s}=x_{n-1}\right\}\right)=1$.

We shall deal only with regular BAD processes. The right boundary will always be $\infty$ (i.e. $\sup S=\infty$ ), the left boundary either 0 or $-\infty$. Feller (1959) showed that we may describe such processes by a speed and scale function $m, p$ plus some boundary conditions analogous to the above for diffusions, which provide an infinite lifetime of the process. An important construction is due to Stone (1963) (see also Dorea and Rosenkrantz (1980) and Kurtz (1969)):

If a sequence $m^{n}, p^{n}$ of speed, scale functions converge to the speed, scale functions $m, p$ pointwise for every continuity point of $m, p$ and the boundaries are $\pm \infty$ and natural, then there are versions of the corresponding sequence $\left(X_{t}^{n}\right)$ of diffusions or BAD processes converging in probability to the corresponding diffusion or BAD process in the Skorohod topology on $D[0, T]$ for all $T>0$.

For a description of the Skorohod topology fix some $T>0 . D[0, T]$ is the space of all functions from $[0, T]$ to $\mathbb{R}$, right continuous with left limits. Define a distance $D_{T}(f, g)$ on $D[0, T]$ as the smallest $\varepsilon>0$ such that there is a continuous, strictly increasing function $M:[0, T] \rightarrow[0, T], M(0)=0, M(T)=T$ with

$$
|M(t)-t|<\varepsilon, \quad|f(t)-g(M(t))|<\varepsilon
$$

for all $t \in[0, T]$. With this metric $D[0, T]$ is a separable metric space, endowed with the Skorohod topology. 
Denote by $\tau_{(a, b)}$ the first leaving time of the interval $(a, b)$ :

$$
\tau_{(a, b)}=\inf \left\{t \geqq 0: X_{t} \notin(a, b)\right\}
$$

Proposition 3.1. Let $\left(X_{t}^{n}\right), n \in \mathbb{N}$, be a sequence of diffusions or BAD processes, $\left(X_{t}\right)$ a diffusion such that for every $T>0$ the Skorohod-distance $D_{T}\left(X_{t}^{n}, X_{t}\right) \underset{n}{\longrightarrow} 0$ in probability. Let $\left(X_{t}\right)$ start in $x \in(a, b)$. Then for all $\{a, b\}$ up to countably many the corresponding passage times $\tau_{(a, b)}^{n}$ converge in distribution to $\tau_{(a, b)}$ as $n \rightarrow \infty$.

Proof. Choose $a, b$ such that $0 \leqq \tau_{(a-\varepsilon, b+\varepsilon)}-\tau_{(a+\varepsilon, b-\varepsilon)} \rightarrow 0$ in probability, as $\varepsilon \rightarrow 0$. Fix $T>0, \varepsilon>0$ so small that $x \in(a+\varepsilon, b-\varepsilon)$. If $n$ is large enough, $D_{T}\left(X_{t}^{n}, X_{t}\right)<\varepsilon$ with probability of at least $1-\varepsilon$. On the event $\left\{D_{T}\left(X_{t}^{n}, X_{t}\right)<\varepsilon\right\}$

$$
\tau_{(a+\varepsilon, b-\varepsilon)} \wedge T-\varepsilon \leqq \tau_{(a, b)}^{n} \wedge T \leqq \tau_{(a-\varepsilon, b+\varepsilon)} \wedge T+\varepsilon .
$$

$a \wedge b$ denotes the minimum of $a$ and $b$. Letting $n \rightarrow \infty$ and then $\varepsilon \rightarrow 0$, in distribution for all $T>0$

$$
\tau_{(a, b)}^{n} \wedge T \rightarrow \tau_{(a, b)} \wedge T
$$

which is enough to proof our claim.

Proposition 3.2. Let $X_{t}$ be a $B A D$ process on $\mathbb{N}=\{0,1,2, \ldots\}, X_{0} \equiv 0$. Then the distribution of $\tau_{n}$ is a convolution of $n$ exponentials.

Proof. This is well-known, e.g. Karlin and McGregor (1959) or Keilson (1971). A quick proof goes as follows: Let $\lambda_{k}, \mu_{k} \geqq 0$ be the transition rates, $k \geqq 0$, i.e.

$$
P_{k}\left(X_{t}=k+1\right)=\lambda_{k} t+o(t), \quad P_{k}\left(X_{t}=k-1\right)=\mu_{k} t+o(t),
$$

as $t \rightarrow 0$. Let $m$ be the initial state and fix some $n>m$. Decomposing $\tau_{n}$ into the time $\rho$ of departure from $m$ and $\tau_{n}-\rho$, by the strong Markov property, for $t \geqq 0$

$$
\begin{aligned}
E_{m}\left(e^{-t \tau_{n}}\right) & =E_{m}\left(e^{-t \rho}\right)\left[P_{m}\left(X_{\rho}=m-1\right) E_{m-1}\left(e^{-t \tau_{n}}\right)+P_{m}\left(X_{\rho}=m+1\right) E_{m+1}\left(e^{-t \tau_{n}}\right)\right] \\
& =\frac{\mu_{m}}{\lambda_{m}+\mu_{m}+t} E_{m-1}\left(e^{-t \tau_{n}}\right)+\frac{\lambda_{m}}{\lambda_{m}+\mu_{m}+t} E_{m+1}\left(e^{-t \tau_{n}}\right)
\end{aligned}
$$

Further $E_{0}\left(e^{-t \tau_{m}}\right) E_{m}\left(e^{-t \tau_{n}}\right)=E_{0}\left(e^{-t \tau_{n}}\right)$, thus denoting $Q_{m}(-t)=1 / E_{0}\left(e^{-t \tau_{m}}\right)$, we get

$$
Q_{m}(t)\left(\lambda_{m}+\mu_{m}-t\right)=\mu_{m} Q_{m-1}(t)+\lambda_{m} Q_{m+1}(t)
$$

$m \geqq 0, Q_{0} \equiv 1, Q_{-1} \equiv 0$. Now the restriction $m<n$ is no longer required. It is obvious by induction on $m$ that $Q_{m}(t)$ is a polynomial of degree $m$. Also by induction $Q_{m}(t)$ has $n$ real roots and between two successive roots of $Q_{n}(t)$ there is one root of $Q_{m-1}(t)$. Obviously by the above probabilistic interpretation all roots have to be positive.

Theorem 3.3. Let $X_{t}$ be a diffusion on $\mathbb{R}^{+}, 0$ reflective, $X_{0} \equiv 0$. Then $\tau_{y}, y>0$, is in distribution the convolution of exponentials. The exponential parameters are all positive. 
Proof. Approximate the diffusion by a sequence of BAD processes in the Skorohod topology and apply Theorem 2.2 and Propositions 3.1, 3.2.

From Corollary 2.6 and 2.10 we get for

$$
\tau_{y}^{*}=\frac{\tau_{y}-E \tau_{y}}{\sqrt{\operatorname{Var} \tau_{y}}}
$$

Theorem 3.4. Under the assumption of Theorem 3.3:

(i) $\tau_{y}^{*}$ is asymptotically normal, iff $E_{0}\left(\tau_{y}^{*}\right)^{3} \underset{y}{\longrightarrow} 0$.

(ii) $\tau_{y}^{*}$ is asymptotically exponential, iff $E_{0}\left(\tau_{y}^{*}\right)^{3} \underset{y}{\longrightarrow} 2$.

Further from Proposition 2.4

Corollary 3.5. Under the assumptions of Theorem 3.3

$$
\operatorname{Var}_{0} \tau_{y} \leqq\left(E_{0} \tau_{y}\right)^{2}, \quad 0 \leqq E\left(\tau_{y}^{*}\right)^{3} \leqq 2 .
$$

Similar inequalities hold for higher moments.

For an analytic approach to Theorem 3.3 and additional information compare, Kac and Krein (1968) and Kent (1980). Kent shows that under more general assumptions concerning the boundary behaviour and starting point in distribution

$$
\tau_{y}=\sum_{k=1}^{\infty} \eta_{k} X_{k}
$$

where $X_{k}$ are independent, exponentially distributed random variables and $\eta_{k}$ are independent, $0-1$-valued, and also independent from $X_{k}$. For our purposes this representation with randomized exponentials is of little use. An analogue to Proposition 2.1 is no longer true, also Theorem 2.2 has no counterpart. The set of weak limits, obtained from convolutions of randomized exponentials, the so-called Bondesson class of distributions is much vaster (cf. Kent (1980) and the literature cited therein).

For the Bessel process Kent (1980) has calculated the exponential parameters. We do this for the Brownian motion with drift.

Example. Let $W_{t}, t \geqq 0$, be a standard Wiener process, $b \in \mathbb{R}$.

We study $X_{t}=\left|W_{t}+b t \cdot \operatorname{sign} W_{t}\right|$. Consider the Laplace transform

$$
\varphi_{a b}(\lambda)=E_{a} \exp \left(-\lambda \tau_{b}\right) .
$$

It is well-known that $\varphi_{x b}(\lambda), 0 \leqq x \leqq b$, solves uniquely the differential equation $L \varphi=\lambda \varphi$, with boundary conditions $\varphi(b)=1, \varphi^{\prime}(0)=0$. Here $L \varphi=\frac{1}{2} \frac{d^{2}}{d x^{2}} \varphi$ $+b \frac{d}{d x} \varphi$. Using $\varphi_{0 x}(\lambda) \varphi_{x b}(\lambda)=\varphi_{0 b}(\lambda), 0 \leqq x \leqq b$, one gets that $1 / \varphi_{0 x}(\lambda), x \geqq 0$, is the solution of $L \varphi=\lambda \varphi$ with initial values $\varphi(0)=1, \varphi^{\prime}(0)=0$. Calculation 
shows that

with

$$
1 / \varphi_{0 x}(\lambda)=h_{x}(\lambda)
$$

where

$$
h_{x}(\lambda)=\frac{1}{2} e^{-b x}\left(\left(1+\frac{b}{c}\right) e^{c x}+\left(1-\frac{b}{c}\right) e^{-c x}\right)
$$

$$
c=c_{\lambda}=\sqrt{2 \lambda+b^{2}} .
$$

By choosing the right branch of the square root function we get a holomorphic continuation of $c_{\lambda}$ and thus $h_{x}(\lambda)$ on $\mathbb{C}-(-\infty, 0] .\left(h_{x}(\lambda)\right.$ is now viewed as a function in $\lambda$ !) It is easily checked that $h_{x}(\lambda)$ may be further extended to a continuous (and thus holomorphic) function on the whole complex plane. On the other hand from Theorem 3.3

$$
\varphi_{0 x}(\lambda)=\prod_{k=1}^{\infty}\left(1+\mu_{k} \lambda\right)^{-1},
$$

where $\mu_{1} \geqq \mu_{2} \geqq \ldots$ are the exponential parameters. Therefore $-1 / \mu_{1},-1 / \mu_{2}$, $\ldots$ are exactly the zeros of $h_{x}(\lambda)$, which all have to be located on $(-\infty, 0)$. Easy calculations show that

$$
\mu_{k}=\frac{2 x^{2}}{s_{k}^{2}+(b x)^{2}}
$$

where $0<s_{1}<s_{2}<\ldots$ are the solutions of the equation

$$
\tan s=-\frac{s}{b x}
$$

If $b x \leqq-1$, one has an additional exponential with parameter

$$
\mu_{0}=\frac{2 x^{2}}{(b x)^{2}-s_{0}^{2}},
$$

where $s_{0}=0$, if $b x=-1$, and $s_{0}$ solves

$$
\text { Tanh } s=-\frac{s}{b x}, \quad s>0,
$$

if $b x<-1$.

\section{Convergence of the First Passage Time}

Let $X_{t}$ be a diffusion. We study the asymptotic behaviour of $\tau_{y}$, as $y \rightarrow \infty$. We shall continue to assume that 0 is a reflecting boundary, but one should note that other situations may be reduced to this case. For example let us consider the case that $X_{t}$ is a diffusion on the whole real line and $X_{t} \rightarrow \infty$, as $t \rightarrow \infty$, with probability 1 . The asymptotic behaviour of $\tau_{y}$ does not change, if we start from a state $x>0$ instead from 0 , since both hitting times differ only by the 
amount $\tau_{x}$. Furthermore 0 will be reached with arbitrarily small probability, if $x$ is large enough. Therefore the asymptotic behaviour of $\tau_{y}$ is not affected, if we make 0 into a reflecting boundary. The reader will have no difficulty to make this argument firm. Of course the change in $E \tau_{y}$ or $\operatorname{Var} \tau_{y}$ might not be neglectible, so one has to take care and use the correct normalization.

Thus in the sequel let 0 be a reflecting and $\infty$ a natural boundary. Further we assume that $X_{t}$ has a smooth infinitesimal operator of the form

$$
L=D_{m} D_{p}^{+}=a D_{x x}+b D_{x},
$$

where $a$ and $b$ are continuous functions, $a>0$ everywhere. ( $D_{x}$ denotes derivation with respect to $x$.) The connection between $a, b$ and $D_{m}, D_{p}^{+}$is given by

$$
D_{m}=\frac{a}{s} D_{x}, \quad D_{p}^{+}=s D_{x} \operatorname{rspl} . d m=\frac{s}{a} d x, \quad d p=\frac{1}{s} d x,
$$

where the function $s>0$ is given by

$$
s(x)=\exp \int_{0}^{x} \frac{b(y)}{a(y)} d y .
$$

The following proposition enables us to calculate moments.

Proposition 4.1. It holds

$$
\begin{aligned}
E_{0} \tau_{y} & =\int_{0}^{y} \int_{0}^{z} d m(u) d p(z), \\
\operatorname{Var}_{0} \tau_{y} & =\int_{0}^{y} \int_{0}^{z} 2 a(u)\left(D_{u} E_{0} \tau_{u}\right)^{2} d m(u) d p(z), \\
E_{0}\left(\tau_{y}-E_{0} \tau_{y}\right)^{3} & =\int_{0}^{y} \int_{0}^{z} 6 a(u)\left(D_{u} E_{0} \tau_{u}\right)\left(D_{u} \operatorname{Var}_{0} \tau_{u}\right) d m(u) d p(z) .
\end{aligned}
$$

Proof. From Mandl (1968), p. 112, for $n \geqq 1$

$$
E_{0} \tau_{y}^{n}=n \int_{0}^{y} \int_{0}^{z} E_{u}\left(\tau_{y}^{n-1}\right) d m(u) d p(z)
$$

Using $E_{u} \tau_{y}=E_{0} \tau_{y}-E_{0} \tau_{u}$ and

$$
E_{u}\left(\tau_{y}^{2}\right)=\operatorname{Var}_{0} \tau_{y}-\operatorname{Var}_{0} \tau_{u}+\left(E_{0} \tau_{y}-E_{0} \tau_{u}\right)^{2},
$$

if $0 \leqq u \leqq y$, some calculations provide the above.

Let again

$$
\tau_{y}^{*}=\frac{\tau_{y}-E_{0} \tau_{y}}{\sqrt{\operatorname{Var}_{0} \tau_{y}}}
$$

An easy situation is considered in the following proposition. 
Proposition 4.2. If $\lim _{y \rightarrow \infty} \operatorname{Var}_{0} \tau_{y}<\infty$, then $\mathbf{L}\left(\tau_{y}^{*}\right)$ converges to a non-normal limit law.

Proof. $\tau_{n}-E_{0} \tau_{n}=\sum_{k=1}^{n} \xi_{k}-E \xi_{k}$, where $\xi_{k}=\tau_{k}-\tau_{k-1}$ are independent random variables. By assumption $\xi=\sum_{k=1}^{\infty} \xi_{k}-E \xi_{k}$ converges a.s. Since $\xi_{1}-E \xi_{1}$ is not normal, the same holds for $\xi$ by a well-known theorem of Cramér.

To have a normal limit of $\tau_{y}^{*}$, one must have $\lim \operatorname{Var}_{0} \tau_{y}=\infty$ as well as $\sqrt{\operatorname{Var}_{0} \tau_{y}}=o\left(E_{0} \tau_{y}\right)$ in view of Corollary 2.8. A partial converse provides the following result.

Proposition 4.3. Assume for $x \rightarrow \infty$

$$
\begin{gathered}
D_{x} \sqrt{\operatorname{Var}_{0} \tau_{x}}=o\left(D_{x} E_{0} \tau_{x}\right), \\
\operatorname{Var}_{0} \tau_{x} \rightarrow \infty, \\
\sqrt{\operatorname{Var}_{0} \tau_{x}} D_{p}^{+} \operatorname{Var}_{0} \tau_{x} \rightarrow \infty
\end{gathered}
$$

Then $\tau_{y}^{*} \rightarrow \mathbf{N}(0,1)$ in distribution, as $y \rightarrow \infty$.

Proof. Using Proposition 4.1, some calculations show

$$
\frac{L E_{0}\left(\tau_{x}-E_{0} \tau_{x}\right)^{3}}{L \operatorname{Var}_{0}^{3 / 2} \tau_{x}}=\frac{4\left(D_{x} E_{0} \tau_{x}\right)\left(D_{x} \sqrt{\operatorname{Var}_{0} \tau_{x}}\right)}{\left(D_{x} E_{0} \tau_{x}\right)^{2}+\left(D_{x} \sqrt{\operatorname{Var}_{0} \tau_{x}}\right)^{2}}
$$

This converges to 0 by the above conditions. Now

$$
\frac{D_{p} E_{0}\left(\tau_{x}-E_{0} \tau_{x}\right)^{3}}{D_{p} \operatorname{Var}_{0}^{3 / 2} \tau_{x}}=\frac{\int_{0}^{x} \frac{L E_{0}\left(\tau_{y}-E_{0} \tau_{y}\right)^{3}}{L \operatorname{Var}_{0}^{3 / 2} \tau_{y}} L \operatorname{Var}_{0}^{3 / 2} \tau_{y} d m(y)}{\int_{0}^{x} L \operatorname{Var}_{0}^{3 / 2} \tau_{y} d m(y)}
$$

converges to 0 by assumption. Finally

$$
\frac{E_{0}\left(\tau_{x}-E_{0} \tau_{x}\right)^{3}}{\operatorname{Var}_{0}^{3 / 2} \tau_{x}}=\frac{\int_{0}^{x} \frac{D_{p} E_{0}\left(\tau_{0}-E_{0} \tau_{y}\right)^{3}}{D_{p} \operatorname{Var}_{0}^{3 / 2} \tau_{y}} D_{p} \operatorname{Var}_{0}^{3 / 2} \tau_{y} d p(y)}{\operatorname{Var}_{0}^{3 / 2} \tau_{x}}
$$

goes to zero by assumption. Now apply Corollary 2.6.

By the same sort of argument one gets 
Proposition 4.4. If one of the conditions

$$
\begin{aligned}
& 0<\frac{\lim }{x} \frac{D_{x} \sqrt{\operatorname{Var}_{0} \tau_{x}}}{D_{x} E_{0} \tau_{x}}, \\
& \varlimsup_{x} D_{p} \operatorname{Var}_{0}^{3 / 2} \tau_{x}<\infty, \\
& \lim _{x} \operatorname{Var}_{0} \tau_{x}<\infty
\end{aligned}
$$

holds, then $\tau_{y}^{*}$ does not converge to a normal distribution.

Remarks. 1. We conjecture that the assumptions of Proposition 4.3 can be further weakened. In fact we believe that $\operatorname{Var}_{0} \tau_{x} \rightarrow \infty$ and $\operatorname{Var}_{0} \tau_{x}=o\left(\left(E_{0} \tau_{x}\right)^{2}\right)$, as $x \rightarrow \infty$, are necessary and sufficient for $\tau_{x}^{*}$ to be asymptotically normal. For the exponential case we have Corollary 2.11 instead of Proposition 4.3. Again we believe that in the case of hitting time the condition formulated in Corollary 2.11 is both necessary and sufficient. Finally Proposition 4.4 holds for the exponential instead for the normal distribution, if the first condition is replaced by $\varlimsup_{x} D_{x} \sqrt{\operatorname{Var}_{0} \tau_{x}} / D_{x} E_{0} \tau_{x}<1$.

2. Propositions 4.3 and 4.4 may be extended to hold for general (non smooth) diffusions. Here one has to carry out a careful, uniform approximation procedure via smooth diffusions.

Examples. A. The positive recurrent case. It is well-known that the diffusion is positive recurrent, iff

$$
\int_{0}^{\infty} \frac{s(x)}{a(x)} d x=\int_{0}^{\infty} d m=m(\infty)<\infty
$$

Claim. If $m(\infty)<\infty$, then $\tau_{y}^{*}$ is asymptotically exponential.

Proof. $\infty$ is a natural boundary, thus the case $\lim E_{0} \tau_{y}<\infty$ of explosion is excluded. Then from Proposition 4.1

and

$$
E_{0} \tau_{x}=\int_{0}^{x}\left(\int_{0}^{y} d m\right) \frac{1}{s(y)} d y \sim m(\infty) \int_{0}^{x} \frac{d y}{s(y)},
$$

$$
\operatorname{Var}_{0} \tau_{x} \sim \int_{0}^{x} \frac{1}{s(y)} \int_{0}^{y} \frac{2 m(\infty)^{2}}{s(z)} d z d y=m(\infty)^{2}\left(\int_{0}^{x} \frac{d y}{s(y)}\right)^{2} \sim\left(E_{0} \tau_{x}\right)^{2}
$$

Now apply Corollary 2.11 .

This result is closely related to Mandl (1968), p. 102, Theorem 1. 
B. The case $a \equiv 1 . a \equiv 1$ is not an essential restriction, since by a change of scale every diffusion with smooth coefficients can be transformed to this situation.

Claim. If $a \equiv 1, b>0$ everywhere, $b^{\prime}(x)=o\left(b^{2}(x)\right)$, as $x \rightarrow \infty$, and $\int_{0}^{\infty} b(x)^{-3} d x=\infty$, then $\tau_{y}^{*}$ is asymptotically $\mathbf{N}(0,1)$-distributed.

Proof. From $b^{\prime} b^{-2}=o(1)$ we get $b(x)^{-1}=o(x)$, thus $s(x) \rightarrow \infty$. Also, if $n \geqq 0$, for large $x$

therefore

$$
\begin{aligned}
b(x)^{-n} s(x) & \geqq b(x)^{-n} \exp \left(\frac{1}{2} \int_{0}^{x} b\right) \exp \left(n \int_{0}^{x} \frac{b^{\prime}}{b}\right) \\
& =b(0)^{-n} \sqrt{s(x)},
\end{aligned}
$$

$$
b(x)^{-n} s(x) \rightarrow \infty, \quad \text { as } \quad x \rightarrow \infty .
$$

By an application of l'Hospitals rule

$$
\int_{0}^{x} s(y) b(y)^{-n} d y \sim s(x) b(x)^{-n-1}, \quad x \rightarrow \infty .
$$

Using this and Proposition 4.1, check, as $y \rightarrow \infty$

$$
\begin{aligned}
& E_{0} \tau_{y} \sim \int_{0}^{y} \frac{d z}{b(x)} \\
& \operatorname{Var}_{0} \tau_{y} \sim 2 \int_{0}^{y} \frac{d z}{b(z)^{3}} \\
& E_{0}\left(\tau_{0}-E_{0} \tau_{y}\right)^{3} \sim 12 \int_{0}^{y} \frac{d z}{b(z)^{5}} .
\end{aligned}
$$

Now

$$
b^{-4}(x)=O\left(\int_{0}^{x} b^{\prime} b^{-5}\right)=o\left(\int_{0}^{x} b^{-3}\right)
$$

and an application of l'Hospitals rule shows

$$
\frac{E_{0}\left(\tau_{x}-E_{0} \tau_{x}\right)^{3}}{\operatorname{Var}_{0}^{3 / 2}} \sim \frac{12}{2^{3 / 2}} \frac{\int_{0}^{x} b^{-5}}{\left(\int_{0}^{x} b^{-3}\right)^{3 / 2}} \rightarrow 0
$$

Now apply Theorem 3.4 . 
One could also have applied Proposition 4.3 in the last proof. Our result is better than for example Theorem 11 in Mandl (1968), p. 129. There roughly $0<c \leqq b(x) \leqq x^{1 / 3-\varepsilon}$ is needed. Here we essentially require $x^{-1} \ll b(x) \leqq x^{1 / 3}$. (A similar result can be found in Keller et al. (1984), but here we need much less regularity.) Our result is close to the best possible. The condition $\int_{0}^{\infty} b^{-3}=\infty$ cannot be relaxed, since it ensures $\operatorname{Var} \tau_{y} \rightarrow \infty$ as $y \rightarrow \infty$. The transient OrnsteinUhlenbeck process $\left(X_{t}\right)$ (with reflection at 0 ) may serve as an example. Here

$$
L=\frac{1}{2} D_{x x}+x D_{x} .
$$

It is well-known that $X_{t}$ has the representation $X_{t}=e^{t}\left|W\left(1-e^{-2 t}\right)\right|$, where $\left(W_{t}\right)$ is a Wiener process. Since $e^{-t} X_{t} \rightarrow|W(1)|$, it is fairly obvious that for the corresponding hitting times

$$
\operatorname{Pr}\left(\tau_{x}-\log x \leqq t\right) \rightarrow \operatorname{Pr}\left(|W(1)| \leqq e^{-t}\right) .
$$

Also the condition $b^{\prime}=o\left(b^{2}\right)$ is close to being the optimum. This follows from

Claim. Assume $b(x) \leqq c x^{-1}$ for some $c>0$. Then $\tau_{y}^{*}$ does not converge to a normal distribution.

Proof. We start from the estimate, valid for $y \geqq 2$,

$$
\begin{aligned}
\operatorname{Var}_{0} \tau_{y} & \geqq\left[\frac{\int_{1}^{y}\left(D_{z} E_{0} \tau_{z}\right) \int_{0}^{z} s(u) d u d z^{2}}{\int_{0}^{y} s(u) d u}\right] \\
& \geqq\left[\frac{\int_{y / 2}^{y}\left(D_{z} E_{0} \tau_{z}\right) d z \int_{0}^{y / 2} s(u) d u^{2}}{\int_{0}^{y} s(u) d u}\right]
\end{aligned}
$$

For a proof of the first inequality take derivatives on both sides and apply Proposition 4.1. Next note that

$$
s(x) x^{-c}=s(1) \exp \left(\int_{1}^{x}\left(b(z)-\frac{c}{z}\right) d z\right)
$$

is a decreasing function. Therefore

$$
\int_{0}^{y} s\left|\int_{0}^{y / 2} s=1+\int_{y / 2}^{y} s\right| \int_{0}^{y / 2} s \leqq 1+\int_{y / 2}^{y} z^{c} d z / \int_{0}^{y / 2} z^{c} d z=2^{c+1} .
$$

Combining the estimates we get

$$
\operatorname{Var}_{0} \tau_{y} \geqq 2^{-2 c-2}\left(E_{0} \tau_{y}-E_{0} \tau_{y / 2}\right)^{2} .
$$


Suppose now that $\tau_{y}^{*}$ converges to a normal. By Corollary $2.8 \operatorname{Var}_{0} \tau_{y}=o\left(\left(E_{0} \tau_{y}\right)^{2}\right)$ and we conclude that $E_{0} \tau_{y}$ is slowly varying in $y$. Especially

$$
E_{0} \tau_{y}=o(y) \text {. }
$$

On the other hand for $\gamma>0$ small enough $s(x) \geqq \gamma s(x) b(x)$ for all $x$. By an integration $\int_{0}^{z} s(x) d x \geqq \gamma(s(z)-s(0))$, thus $\int_{0}^{z} s(x) d x \geqq \frac{\gamma}{2} s(z)$ for large $z$, if $\gamma$ was chosen small enough. By Proposition 4.1 we derive the contradiction

$$
E_{0} \tau_{y}=\int_{0}^{y} s(z)^{-1} \int_{0}^{z} s(x) d x d z \geqq \frac{\gamma}{2} y+\text { const }
$$

for all $y$.

\section{References}

Dorea, C., Rosenkrantz, W.: Limit theorems for Markov processes via a variant of the Trotter-Kato Theorem. J. Appl. Probab. 17, 704-715 (1980)

Feller, W.: The birth-and-death process as a diffusion process. J. Math. Pures Appl. 38, 301-345 (1959)

Kac, I.S., Krein, M.G.: O spektralnych funkcii struny. Appendix to the Russian translation of Atkinson, Discrete and continuous boundary problems. Moskva: Mir. (1968)

Karlin, S.: Total positivity. Stanford University Press 1968

Karlin, S., McGregor, J.: Coincidence properties of birth and death processes. Pac. J. Math. 9, 11091140 (1959)

Keilson, J.: Log-concavity and log-convexity in passage time densities of diffusion and birth-death processes. J. Appl. Probab. 8, 391-398 (1971)

Keller, G., Kersting, G., Rösler, U.: On the asymptotic behaviour of solutions of stochastic differential equations. Z. Wahrscheinlichkeitstheor. Verw. Geb. 68, 163-189 (1984)

Kent, J.T.: Eigenvalue expansions for diffusion hitting times. Z. Wahrscheinlichkeitstheor. Verw. Geb. 52, 309-319 (1980)

Kurtz, Th.: Extension of Trotter's operator semigroup approximation theorems. J. Funct. Anal. 3, 354-375 (1969)

Mandl, P.: Analytical treatment of one-dimensional Markov-processes. Berlin Heidelberg New York: Springer 1968

Stone, C.J.: Limit theorems for random walks, birth and death processes and diffusion processes. Illinois J. Math. 7, 638-660 (1963)

Received January 11, 1987 